\title{
ESC-Leitlinie 2015: Diagnostik und Therapie der pulmonalen Hypertonie
}

Christian Opitz, Stephan Rosenkranz, Hossein Ardeschir Ghofrani, Ekkehard Grünig, Hans Klose, Horst Olschewski, Marius Hoeper

\section{Was ist neu?}

- Definition und Klassifikation: Die Definition der pulmonal arteriellen Hypertonie (PAH) berücksichtigt nun den pulmonal-vaskulären Widerstand (>3 Wood Einheiten). Geringe Veränderungen der Klassifikation in den Gruppen $1-5$.

- Diagnostik: Zunehmende Bedeutung gewinnt die Diagnostik und Therapie von Patienten mit PH der Gruppen 2 und 3.

- Therapie: Die Therapie der PH orientiert sich nun am abgeschätzten 1-Jahres-Überleben, nicht mehr an einzelnen „Zielwerten“. Die Kombinationstherapie kommt früher zum Einsatz. Mehrere neue Substanzen stehen für die gezielte Therapie der PH zur Verfügung.

- PH bei Linksherzerkrankungen: Die post-kapilläre pulmonalen Hypertonie (PH) wird unterteilt in isolierte und kombinierte Formen. Im Vordergrund steht weiterhin die optimierte Therapie der Linksherzerkrankung.

- PH bei Lungenerkrankungen: Als schwere Formen der PH bei Lungenerkrankungen wurden definiert: mittlerer pulmonal arterieller Druck (PAPm) $\geq 35 \mathrm{mmHg}$ oder die Kombination aus PAPm $\geq 25 \mathrm{mmHg}$ und Herzindex $<2,5 \mathrm{I} / \mathrm{min} / \mathrm{m}^{2}$. Im Vordergrund steht weiterhin die optimierte Therapie der Lungenerkrankung.

- Chronisch thromboembolische PH: Neu definiert wird das Krankheitsbild der chronisch thromboembolischen Lungenerkrankung (CTED), bei der die Kriterien einer CTEPH erfüllt sind, der PAPm aber noch unter 25 mmHg liegt. Riociguat wurde als erste Substanz für die Therapie der nichtoperablen oder rezidivierenden chronisch thromboembolischen $\mathrm{PH}$ zugelassen. Therapie der Wahl ist weiterhin die pulmonale Endarteriektomie, für ungeeignete Patienten wird die Ballonangioplastie evaluiert.

Tab. 1 Klinische Klassifikation der pulmonalen Hypertonie (PH).

1. Pulmonal arterielle Hypertonie (PAH)

1.1 Idiopathisch (IPAH)

1.2 Hereditär (HPAH)

1.3 Durch Medikamente oder Toxine verursacht

1.4 Assoziiert mit:

1.4.1 Bindegewebserkrankungen

1.4.2 Infektion mit dem Humanen Immundefizienz-Virus (HIV)

1.4.3 Portaler Hypertension

1.4.4 Angeborenen Herzfehlern

1.4.5 Schistosomiasis

1' Pulmonale veno-okklusive Erkrankung und / oder pulmonale kapilläre Hämangiomatose

1" Persistierende pulmonale Hypertonie des Neugeborenen

2. Pulmonale Hypertonie infolge von Linksherzerkrankungen

3. Pulmonale Hypertonie infolge von Lungenerkrankungen und/oder Hypoxie

4. Chronisch thromboembolische pulmonale Hypertonie (CTEPH) und andere Pulmonalarterien-Obstruktionen

5. Pulmonale Hypertonie mit unklarem und / oder multifaktoriellem Mechanismus

\section{Glossar}

CTEPH = chronisch thromboembolische pulmonale Hypertonie

PAH = pulmonal arterielle Hypertonie

PAPm = mittlerer pulmonal arterieller Druck

PAPsys $=$ systolischer pulmonal arterieller Druck

$\mathbf{P A W P}=$ pulmonal arterieller Verschlussdruck

$\mathbf{P H}=$ pulmonale Hypertonie

PVR= pulmonal-vaskulärer Widerstand

$\mathbf{T P G}=$ transpulmonaler Gradient

\section{Aktueller Stand}

Die pulmonale Hypertonie (PH) ist durch den Anstieg des (invasiv bestimmten) mittleren pulmonal arteriellen Drucks (PAPm) auf $\geq 25 \mathrm{mmHg}$ in Ruhe definiert (Normalwert $14 \pm 3 \mathrm{mmHg}$ ). Sie geht häufig mit einer erheblichen Einschränkung der Lebensqualität und Prognose einher. Ursache sind meist vaskuläre Veränderungen der Lunge, welche eigenständig oder in Assoziation mit anderen Erkrankungen auftreten können. Letztlich führen diese Erkrankungen über eine Belastung bzw. das Versagen des rechten Herzens zum Tod.

In den letzten Jahren konnten wesentliche Fortschritte in der Diagnostik und Therapie der PH, insbesondere der pulmonal arteriellen Hypertonie (PAH), erzielt werden. Diese wurden bei der Aktualisierung der gemeinsamen Leitlinien der European Society of Cardiology (ESC) und der European Respiratory Society (ERS) zur Diagnostik und Therapie der pulmonalen Hypertonie im Jahr 2015 berücksichtigt [1]. Neu sind Änderungen der hämodynamischen Definitionen, der Empfehlungen zum diagnostischen Vorgehen bei Verdacht auf PH sowie zur Risikostratifizierung und Therapie der PAH. Schließlich beinhalten die neuen ESC/ERS-Leitlinien detaillierte Empfehlungen zum Management anderer PH-Formen wie der PH bei Linksherz- oder Lungenerkrankungen und der chronisch thromboembolischen pulmonalen Hypertonie und definieren Kriterien für spezialisierte Expertenzentren.

\section{Definition und Klassifikation}

Abgesehen von geringen Anpassungen teilen die neuen Leitlinien die PH unverändert in 5 Gruppen ein, welche sich an Gemeinsamkeiten in Pathophysiologie und Therapie orientieren $(\bullet$ Tab. 1$)$. 
Hämodynamisch wird weiterhin mit Hilfe des pulmonal arteriellen Verschlussdruckes (PAWP) unterschieden zwischen

- prä-kapillärer PH: PAWP $\geq 15$ mmHg, Gruppen 1, 3, 4 und 5 und

- post-kapillärer PH: PAWP > 15 mmHg, Gruppe 2 Neu in der hämodynamischen Definition der pulmonal arteriellen Hypertonie (PAH) ist das Erfordernis eines pulmonalvaskulären Widerstandes (PVR) von > 3 Wood-Einheiten. Damit findet neben dem Druckniveau die Veränderung des Herzzeitvolumens besondere Berücksichtigung.

Am häufigsten tritt die $\mathrm{PH}$ in Assoziation mit Linksherz- oder Lungenerkrankungen auf, während die PAH weiterhin selten ist. Die CTEPH wird vermutlich immer noch in ihrer Häufigkeit unterschätzt bzw. nicht ausreichend diagnostiziert.

\section{Klinische Relevanz \\ Unverändert ist die korrekte Klassifikation der \\ $\mathrm{PH}$ die entscheidende Grundlage für eine adäquate Therapie und sollte in Zusammenar- beit mit einem PH-Expertenzentrum erfolgen.}

\section{Diagnostik}

Die Symptome der PH sind häufig unspezifisch wie

- Dyspnoe,

- Müdigkeit,

- körperliche Schwäche,

- Angina pectoris,

- trockener Husten und

- Synkopen.

Die Diagnose erfordert daher den klinischen Verdacht sowie die Durchführung gezielter Untersuchungen $(\triangleright$ Tab. 2$)$.

Zentrale Bedeutung besitzt hier die Echokardiografie, welche häufig eine erste Abschätzung des systolischen PAP (PAPsys) sowie eine Beurteilung des rechten Herzens erlaubt. Ein abgeschätzter PAPsys von $>50 \mathrm{mmHg}$ spricht für eine hohe $\mathrm{PH}-$ Wahrscheinlichkeit und sollte weiter abgeklärt werden, einschließlich Rechtsherzkatheter-Untersuchung (I, C). Werte $>35 \mathrm{mmHg}$ sollten beim Vorliegen von PH-Risikofaktoren (z.B. Sklerodermie), Rechtsherzbelastungszeichen oder unklarer Dyspnoe ebenfalls weiter abgeklärt werden (IIa, B).

Im nächsten Schritt wird beurteilt, ob Linksherzoder Lungenerkrankungen als häufigste Ursachen einer PH in Betracht kommen. Wenn ja, steht die kardiologische bzw. pneumologische Abklärung zunächst im Vordergrund. Wenn dies nicht der Fall ist, sollte die weitere Abklärung in Kooperation mit einem PH-Expertenzentrum erfolgen.
Bei jeder schweren PH ist die Ventilations- / Perfusions-Szintigrafie Bestandteil der initialen diagnostischen Abklärung. Sie bleibt die Methode der Wahl zum Ausschluss einer chronisch thromboembolischen PH (CTEPH) und ist der CT-Angiografie überlegen. Zur Bestätigung der Diagnose PH, der ätiologischen Abklärung und der Ermittlung des hämodynamischen Schweregrades ist die Rechtsherzkatheter-Untersuchung (RHK) erforderlich. Auch die Vasoreagibilitäts-Testung (neu: indiziert nur noch bei IPAH, HPAH oder Medikamenten-induzierter PAH) zur Erkennung möglicher Kandidaten für eine Therapie mit Kalziumantagonisten gelingt nur invasiv und sollte Experten-Zentren vorbehalten bleiben (I, C).

Erst die exakte Klassifikation der PH und die Bestimmung des Schweregrades der Erkrankung erlauben eine angemessene und effektive Therapie. Grundlage dafür ist eine angemessene Differenzialdiagnostik, möglichst in Zusammenarbeit mit einem PH-Expertenzentrum, auch um auslösende oder assoziierte Erkrankungen sicher erkennen bzw. ausschließen zu können.

\section{Klinische Relevanz \\ Wichtig bleibt der klinische Verdacht bei insgesamt eher unspezifischen Symptomen. Die Echokardiografie als wichtigste nicht- invasive Methode liefert meist erste Hinweise.}

\section{Therapie}

Neu ist das Konzept der Risikostratifikation als Grundlage für das therapeutische Vorgehen und die prognostische Einschätzung. Basierend auf der zu erwartenden 1-Jahres-Mortalität werden Patienten nach eingehender Untersuchung in solche mit „niedrigem Risiko“ ( $<5 \%)$, „intermediärem Risiko“ (5-10\%) oder „hohem Risiko“ (>10\%) eingestuft. Zur Abschätzung dieses individuellen Risikos werden Symptome, funktionelle Klasse, Belastungstests, Laboruntersuchungen, Echokardiografie und RHK gemeinsam bewertet ( $\bullet$ Tab. 3),

- Elektrokardiogramm

- Röntgen-Thorax

- Echokardiografie

- Lungenfunktionstest und arterielle bzw. kapilläre Blutgas-Analyse

- Ventilations-/Perfusions-Szintigrafie

- Hoch-auflösende Computertomografie mit Kontrastverstärkung

- Kardiale Magnetresonanztomografie

- Blutuntersuchungen inklusive immunologisches Labor

- Abdomen-Ultraschall

- Rechtsherzkatheter (RHK) und ggf. Vasoreagibilitätstestung

- Pulmonalis-Angiografie 
Tab. 3 Abschätzung der 1-Jahres-Mortalität bei Patienten mit pulmonaler Hypertonie [1]. da keine einzelne Variable ausreichend Informationen liefern kann. Als Therapieziel gilt das Erreichen des Status „geringes Risiko“ für möglichst viele Parameter. Diese Risiko-Abschätzung wird auch im Verlauf regelmäßig wiederholt und bei nicht adäquatem Therapieansprechen erfolgt eine entsprechende Therapie-Eskalation.

Die neuen Leitlinien beschreiben eine Weiterentwicklung der PH-Therapie, welche nicht allein durch Medikamente erfolgt, sondern eine komplexe Gesamtstrategie darstellt. Diese umfasst Allgemeinmaßnahmen und supportive Therapien, die Behandlung von vasoreaktiven Patienten mit hochdosierten Kalziumantagonisten sowie den Einsatz gezielter PAH-Medikamente als Mono- oder Kombinationstherapie mit regelmäßiger Beurteilung des Therapieeffektes. Ultima Ratio bleibt die Lungentransplantation.

$\mathrm{Zu}$ den unterstützenden Therapiemaßnahmen gehören z.B.

- Diuretika (I-C),

- Sauerstofflangzeittherapie (wenn PaO2<60 mmHg; I-C),

- Antikoagulation (IIb-C),

- Ausgleich einer Anämie/eines Eisenmangels (IIb-C),
- Influenza- und Pneumokokkenimpfungen (I-C) und

- betreutes körperliches Training in spezialisierten Einrichtungen (IIa-B).

Als Neuerung fällt die o.g. Empfehlung zur Antikoagulation etwas restriktiver aus und gilt nur noch für Patienten mit IPAH, HPAH und durch Medikamente oder Toxine verursachte PAH. Eine effektive Kontrazeption wird unverändert empfohlen, da Schwangerschaft und Entbindung weiterhin mit einem erheblichen Risiko für PHPatientinnen einhergehen. Die effektive Rhythmuskontrolle bei PH-Patienten mit Vorhofflimmern oder Vorhofflattern wird zunehmend als Therapieziel wahrgenommen und ist mit einer günstigeren Prognose assoziiert. Neben der medikamentösen Therapie mit Amiodaron gewinnt hier die interventionelle elektrophysiologische Therapie an Bedeutung.

Für die gezielte Therapie der PAH stehen in Deutschland mittlerweile zahlreiche Medikamente aus verschiedenen Substanzklassen zur Verfügung. Diese umfassen Endothelin-Rezeptor-Antagonisten (ERA; Ambrisentan, Bosentan, Macitentan), Phosphodiesterase-5 Inhibitoren (PDE5i; Sildenafil, Tadalafil), einen sGC-Stimulator (Riociguat) sowie Prostazyklin-Analoga (Epoprostenol, Iloprost, Tre-
Prognoseparameter ${ }^{\mathrm{a}}$

Klinische Zeichen einer Rechtsherzinsuffizienz

Progression der Symptome

Synkope

WHO-Funktionsklasse

6-Minuten-Gehtest

Kardiopulmonaler Belastungstest (Spiroergometrie)

BNP / NTproBNP-

Plasmaspiegel

Bildgebung

(Echokardiografie, cMRT)

\begin{tabular}{|c|}
\hline Geringes Risiko < $5 \%$ \\
\hline nicht vorliegend \\
\hline nein \\
\hline nein \\
\hline ।, II \\
\hline$>440 \mathrm{~m}$ \\
\hline $\begin{array}{l}\text { Höchstwert } \mathrm{VO}_{2}>15 \mathrm{ml} / \mathrm{min} / \\
\mathrm{kg}(>65 \% \text { pred.) } \\
\mathrm{VE} / \mathrm{VCO}_{2} \text {-Slope }<36\end{array}$ \\
\hline $\begin{array}{l}\text { BNP }<50 \mathrm{ng} / \mathrm{l} \\
\text { NTproBNP }<300 \mathrm{ng} / \mathrm{I}\end{array}$ \\
\hline $\begin{array}{l}\text { RA-Fläche }<18 \mathrm{~cm}^{2} \\
\text { kein Perikarderguss }\end{array}$ \\
\hline $\begin{array}{l}\mathrm{RAP}<8 \mathrm{mmHg} \\
\mathrm{Cl} \geq 2,5 \mathrm{I} / \mathrm{min} / \mathrm{m}^{2} \\
\mathrm{SvO}_{2}>65 \%\end{array}$ \\
\hline
\end{tabular}

\begin{tabular}{|c|c|}
\hline Intermediäres Risiko 5-10\% & Hohes Risiko > $10 \%$ \\
\hline nicht vorliegend & vorliegend \\
\hline langsam & schnell \\
\hline gelegentliche Synkopen ${ }^{b}$ & wiederholte Synkopen ${ }^{c}$ \\
\hline III & IV \\
\hline $165-440 m$ & $<165 m$ \\
\hline $\begin{array}{l}\text { Höchstwert } \mathrm{VO}_{2} 11-15 \mathrm{ml} / \\
\mathrm{min} / \mathrm{kg}(35-65 \% \text { pred.) } \\
\text { VE } / \mathrm{VCO}_{2} \text {-Slope } 36-44\end{array}$ & $\begin{array}{l}\text { Höchstwert } \mathrm{VO}_{2}<11 \mathrm{ml} / \mathrm{min} / \\
\mathrm{kg}(<35 \% \text { pred.) } \\
\mathrm{VE} / \mathrm{VCO}_{2} \text {-Slope } \geq 45\end{array}$ \\
\hline $\begin{array}{l}\text { BNP } 50-300 \mathrm{ng} / \mathrm{l} \\
\text { NTproBNP } 300-1400 \mathrm{ng} / \mathrm{I}\end{array}$ & $\begin{array}{l}\text { BNP }>300 \mathrm{ng} / \mathrm{I} \\
\text { NTproBNP }>1400 \mathrm{ng} / \mathrm{I}\end{array}$ \\
\hline $\begin{array}{l}\text { RA-Fläche } 18-26 \mathrm{~cm}^{2} \\
\text { kein oder minimaler Perikard- } \\
\text { erguss }\end{array}$ & $\begin{array}{l}\text { RA-Fläche }>26 \mathrm{~cm}^{2} \\
\text { Perikarderguss }\end{array}$ \\
\hline $\begin{array}{l}\mathrm{RAP} 8-14 \mathrm{mmHg} \\
\mathrm{Cl} 2,0-2,4 \mathrm{I} / \mathrm{min} / \mathrm{m}^{2} \\
\mathrm{SvO}_{2} 60-65 \%\end{array}$ & $\begin{array}{l}\mathrm{RAP}>14 \mathrm{mmHg} \\
\mathrm{Cl}<2,0 \mathrm{I} / \mathrm{min} / \mathrm{m}^{2} \\
\mathrm{SvO}_{2}<60 \%\end{array}$ \\
\hline
\end{tabular}

BNP = Brain Natriuretic Peptide; $\mathrm{Cl}=$ Herzindex; SvO2 = gemischt-venöse Sauerstoffsättigung; NT-proBNP= N-terminales Pro Brain Natriuretic peptide; pred. = vorhergesagt; RA = rechter Vorhof: RAP = rechter Vorhofdruck; VO2 = Sauerstoffaufnahme; VE/VCO2 = Atemäquivalent für Kohlendioxid; $W H O=$ World Health Organization; 6MWT = 6-Minuten-Gehtest.

aDie meisten der vorgeschlagenen Variablen und Höchstwerte basieren auf der Meinung von Experten. Sie können Informationen hinsichtlich der Prognose liefern und können für Therapieentscheidungen verwendet werden, die Anwendung beim einzelnen Patienten muss jedoch mit Bedacht erfolgen.

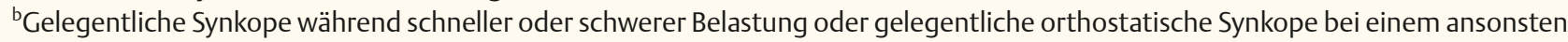
stabilen Patienten.

'Wiederholte Episoden einer Synkope, auch bei geringer oder regelmäßiger körperlicher Aktivität. 
prostinil) bzw. einen Prostazyklin-Rezeptor-Agonisten (Selexipag). Mit Macitentan [2], Riociguat [3] und Selexipag [4] wurden kürzlich 3 neue Substanzen zugelassen, welche in dem neuen Therapiealgorithmus berücksichtigt werden. Riociguat erhielt als erstes Medikament die Zulassung für die Therapie der nicht-operablen oder rezidivierenden CTEPH.

Ebenfalls neu bewertet wurde der Stellenwert einer gezielten Kombinationstherapie: Erstmals konnte in der AMBITION-Studie gezeigt werden, dass eine initiale Kombinationstherapie (Ambrisentan+Tadalafil) bei therapienaiven Patienten mit PAH der jeweiligen Monotherapie überlegen war [5]. Bereits in den Zulassungsstudien für Riociguat, Macitentan und Selexipag fiel auf, dass der primäre Studienendpunkt nicht nur bei behandlungsnaiven Patienten, sondern auch bei solchen, die mit anderen Substanzklassen vorbehandelt waren, signifikant verbessert wurde.

Unverändert haben Kalziumantagonisten nur bei Patienten mit IPAH, HPAH oder Medikamenteninduzierter PAH einen Stellenwert, welche in der o.g. Vasoreagibilitäts-Testung am Expertenzentrum die Responder-Kriterien erfüllen (I-C).

\section{Klinische Relevanz}

Neu sind die Risiko-Orientierung sowie der zunehmende Stellenwert einer frühen

Kombinationstherapie als Teile einer komplexen Gesamtstrategie der PH-Therapie mit mehreren neu zugelassenen Medikamenten.

\section{PH bei Linksherzerkrankungen}

Die PH in Assoziation mit Linksherzerkrankungen (Gruppe 2) ist mit einem Anteil von bis zu $50 \%$ aller Fälle die häufigste Form der PH [6] und ist vor allem bei Patienten mit Herzinsuffizienz untersucht. Neben anamnestischen Angaben und klinischer Präsentation, EKG-Befunden, echokar- diographischen Zeichen und anderen bildgebenden Verfahren können insbesondere Risikofaktoren für Herzerkrankungen (bekannte KHK, arterielle Hypertonie, Diabetes mellitus, Adipositas, Vorhofflimmern, etc.) den Verdacht auf eine Linksherzerkrankung als Ursache der PH lenken.

Diese Patienten haben eine post-kapilläre $\mathrm{PH}$ (PAWP > $15 \mathrm{mmHg}$ ) und pathophysiologisch steht die passive Rückwärts-Übertragung erhöhter Füllungsdrucke in die pulmonale Strombahn im Vordergrund. Allerdings kann diese chronische pulmonal venöse Stauung zusätzliche Komponenten triggern (z.B. Vasokonstriktion und vaskuläres Remodeling), welche zu einem weiteren Druck- und Widerstandsanstieg führen.

Bislang wurde der transpulmonale Gradient (TPG = Differenz zwischen PAPm und PAWP) heranzogen, um eine „passive“ PH (TPG $<12 \mathrm{mmHg}$ ) von einer „reaktiven“ bzw. „out-of-proportion“ $\mathrm{PH}$ (TPG $\geq 12 \mathrm{mmHg}$ ) abzugrenzen. Dieses Ziel wurde in der Praxis jedoch nicht zuverlässig erreicht, so dass die neuen Leitlinien eine neue Sub-Klassifikation einführen: je nach diastolischem Druckgradienten $(\mathrm{DPG}=$ diastolischer PAP-PAWP) und pulmonal vaskulärem Widerstand (PVR) wird nun zwischen isoliert post-kapillärer PH (Ipc-PH) und kombiniert post- und prä-kapillärer $\mathrm{PH}$ (Cpc-PH) unterschieden.

Ob sich diese neue Einteilung in der Praxis bewähren wird, bleibt Gegenstand aktueller Diskussionen, insbesondere die fakultative (und/oder) Einbeziehung des PVR (siehe Beitrag Rosenkranz et. al. in diesem Heft [13]). Bei der Therapie dieser Patienten steht weiterhin die möglichst optimale Therapie der Linksherzerkrankung mit optimaler Nachlastsenkung und Volumenkontrolle im Vordergrund (I, C). Aktuelle Daten weisen jedoch darauf hin, dass dieses Ziel noch nicht häufig genug erreicht wird [7]. Eine gezielte Therapie mit „PAHMedikamenten“ wird für diese Patienten in den Leitlinien weiterhin abgelehnt (III, C), nachdem kontrollierte Studien keine positiven Ergebnisse

$\begin{array}{ll}\text { Definition } & \text { Charakteristika }^{a} \\ \text { Post-kapilläre PH } & \text { PAPm } \geq 25 \mathrm{mmHg} \\ & \text { PAWP }>15 \mathrm{mmHg}\end{array}$

Isoliert post-kapilläre $\mathrm{PH}$

(Ipc-PH)

Kombiniert post-kapilläre und prä-kapilläre $\mathrm{PH}(\mathrm{Cpc}-\mathrm{PH})$

\author{
DPG $<7 \mathrm{mmHg}$ und / oder \\ $\mathrm{PVR} \leq 3 \mathrm{WE}^{\mathrm{C}}$ \\ DPG $\geq 7 \mathrm{mmHg}$ und/ oder \\ $P V R>3 W^{C}$
}

\author{
Klinische Gruppen ${ }^{\text {b }}$ \\ 2. PH infolge von Linksherz- \\ erkrankungen \\ 5. PH mit unklarem und/ oder \\ multifaktoriellem Mechanismus
}

HZV = Herzzeitvolumen, DPG = diastolischer Druckgradient; PAWP = pulmonal arterieller Verschlussdruck; PVR = pulmonal vaskulärer Widerstand; $W E=$ Wood-Einheiten.

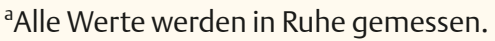

bs. Tab. 1

'Wood-Einheiten werden dyn ${ }^{*} \mathrm{sek}^{*} \mathrm{~cm}^{-5}$ vorgezogen.

Tab. 4 Subklassifikation der pulmonalen Hypertonie. 
zeigten [8-10]. Allenfalls in selektierten Einzelfällen kann eine derartige Therapie probatorisch in enger Abstimmung mit einem PH-Zentrum erfolgen [11], bevorzugt im Rahmen klinischer Studien.

\section{Klinische Relevanz}

Zahlenmäßig wichtigste PH-Gruppe, für die weiterhin die Leitlinien-gerechte Therapie der Herzinsuffizienz im Vordergrund steht. PAH-Medikamente sind hier nicht zugelassen und kommen allenfalls für Einzelfälle im Rahmen von Studien oder an PH-Expertenzentren in Betracht.

\section{PH bei Lungenerkrankungen}

Die zahlenmäßig zweitgrößte PH-Gruppe (ca. 30 -45\%) bilden die Patienten mit Lungenerkrankungen (Gruppe 3). Wie in der Gruppe 2 gilt auch für Patienten mit Lungenerkrankungen, welche eine $\mathrm{PH}$ entwickeln, eine deutlich schlechtere Prognose mit häufigeren Hospitalisationen. Die betroffenen Patienten leiden am häufigsten an einer COPD, einer Lungenfibrose oder CPFE (combined pulmonary fibrosis and emphysema). Während leichte Steigerungen des PAPm (bis $25 \mathrm{mmHg}$ ) bei Patienten mit Lungenerkrankungen sehr häufig sind, bleiben Werte $>40 \mathrm{mmHg}$ selten. Mit dem Ziel schwere Formen der PH bei Patienten mit Lungenerkrankungen zu definieren (welche möglicherweise mit PAH-Medikamenten behandelt werden könnten) wurden als neue Grenzwerte ein PAPm $>35$ mmHg oder die Kombination eines PAP $\geq 25 \mathrm{mmHg}$ und einem Herzindex $<2,5 \mathrm{l} / \mathrm{min} / \mathrm{m}^{2}$ gewählt [1]. Hier besteht eine Diskrepanz zu früheren Empfehlungen [12], die Gegenstand aktueller Diskussionen bleibt (siehe Beitrag Rosenkranz et. al. in diesem Heft [13]).

Diagnostisch wird die Echokardiografie bei vermuteter PH empfohlen (I, C). Für Patienten mit echokardiografischen Zeichen einer schweren $\mathrm{PH}$ oder einer schweren rechtsventrikulären Dysfunktion wird eine Überweisung an ein PH-Expertenzentrum empfohlen (I, C).

Therapeutisch steht weiterhin die optimale Therapie der Lungenerkrankung inklusive Langzeitsauerstofftherapie im Vordergrund (I, C). Für Patienten mit schwerer PH oder schwerer Rechtsherzinsuffizienz sollte die Überweisung an ein PH-Expertenzentrum zur individuell angepassten Therapie erwogen werden (IIa, C). Eine Rechtsherzkatheter-Untersuchung wird bei V.a. PH nicht empfohlen, es sei denn therapeutische Konsequenzen werden erwartet (z.B. Lungentransplantation, alternative Diagnosen wie PAH oder CTEPH, potenzieller Einschluss in eine klinische Studie) (III, C). PAH-Medikamente werden für Patienten mit PH in Folge von Lungenkrankheiten nicht empfohlen (III, C).

\section{Klinische Relevanz}

Zahlenmäßig ebenfalls wichtige PH-Gruppe. Auch hier steht die Leitlinien-gerechte Therapie der Lungenerkrankung im Vordergrund. PAH-Medikamente sind hier nicht zugelassen und kommen allenfalls für einzelne Patienten mit schwerer $\mathrm{PH}$ an $\mathrm{PH}$-Expertenzentren in Betracht.

\section{Chronisch thromboembolische PH}

Eine chronisch thromboembolische pulmonale Hypertonie (CTEPH) besteht bei einem PAPm $\geq 25 \mathrm{mmHg}$, wenn nach mindestens 3-monatiger effektiver Antikoagulation segmentale oder größere Pulmonalarterien durch fibrosierte Thromboembolien obstruiert oder verschlossen sind. Für die Diagnose wird der Nachweis eines Perfusionsdefektes bzw. einer Gefäßläsion mittels Ventilations / Perfusions-Szintigrafie, Computerbzw. MR-Tomografie oder Pulmonalisangiografie gefordert. Neu definiert wird das Krankheitsbild der chronisch thromboembolischen Lungenerkrankung (CTED), bei der die genannten Kriterien erfüllt sind obwohl der PAPm in Ruhe noch unter $25 \mathrm{mmHg}$ liegt.

Der klinische Verdacht einer CTEPH ist besonders wichtig, auch bei Patienten ohne Lungenembolie in der Anamnese. Diagnostisch ist die Ventilations/Perfusions-Szintigrafie die Methode der Wahl und der CT überlegen. Gesichert wird die Diagnose im Rechtsherzkatheter, ggf. ergänzt durch eine Pulmonalisangiografie und CT.

Die Pulmonale Endarteriektomie (PEA) ist die Therapie der Wahl, falls keine schwerwiegenden Kontraindikationen (Komorbiditäten) vorliegen. Die intrahospitale Letalität liegt in spezialisierten Zentren (>50PEAs / Jahr) <3,5\% und die Mehrzahl der Patienten erfährt postoperativ eine deutliche Linderung der Symptome bei häufig nahezu vollständig normalisierter Hämodynamik.

Die supportive medikamentöse Therapie der CTEPH besteht aus Antikoagulanzien und Diuretika sowie Sauerstofflangzeittherapie bei Hypoxämie. Eine lebenslange Antikoagulation wird auch nach PEA empfohlen. Häufig werden bereits neue orale Antikoagulanzien eingesetzt, obwohl es dafür keine Daten zur Effektivität und Sicherheit bei CTEPH gibt. Neu als medikamentöse Therapieoption ist bisher nur Riociguat zur Therapie der inoperablen oder persistierenden/wiederkehrenden CTEPH nach PEA zugelassen. Als Therapieoption für nicht-operable CTEPH-Patienten gewinnt die pulmonale Ballonangioplastie (BPA) an Bedeutung (IIb, C), sollte allerdings ausgewählten Expertenzentren vorbehalten werden. 


\section{Klinische Relevanz}

Bei vermutlich weiterhin hoher Dunkelziffer ist es besonders wichtig diese PH-Ursache zu berücksichtigen und z. B. frühzeitig eine Lungenszintigrafie durchzuführen. Die Therapieoptionen haben sich entscheidend verbessert: an erster Stelle steht die Operation (PEA) aber es stehen auch medikamentöse (Riociguat) und interventionelle Optionen (BPA) zur Verfügung.

\section{Literatur}

1 Galiè N, Humbert M, Vachiery JL et al. 2015 ESC/ ERS Guidelines for the diagnosis and treatment of pulmonary hypertension: The Joint Task Force for the Diagnosis and Treatment of Pulmonary Hypertension of the European Society of Cardiology (ESC) and the European Respiratory Society (ERS): Endorsed by: Association for European Paediatric and Congenital Cardiology (AEPC), International Society for Heart and Lung Transplantation (ISHLT). Eur Heart ] 2016; 37: 67-119

2 Pulido T, Adzerikho I, Channick RN et al. Macitentan and Morbidity and Mortality in Pulmonary Arterial Hypertension. N Engl J Med 2013; 369: 809-818

3 Ghofrani H-A, Galiè N, Grimminger F et al. Riociguat for the treatment of pulmonary arterial hypertension. N Engl J Med 2013; 369: 330 - 340

4 Sitbon O, Channick R, Chin KM et al. Selexipag for the Treatment of Pulmonary Arterial Hypertension. N Engl J Med 2015; 373: 2522 - 2533

5 Galiè N, Barberà JA, Frost AE et al. Initial Use of Ambrisentan plus Tadalafil in Pulmonary Arterial Hypertension. N Engl J Med 2015; 373: $834-844$

6 Hoeper MM, Humbert M, Souza R et al. A global view of pulmonary hypertension. Lancet Respir Med 2016; 4: 306-322

7 Abraham WT, Stevenson LW, Bourge RC et al. Sustained efficacy of pulmonary artery pressure to guide adjustment of chronic heart failure therapy: complete follow-up results from the CHAMPION randomised trial. Lancet 2016; 387: 453-461

8 Redfield MM, Chen HH, Borlaug BA et al. Effect of Phosphodiesterase-5 Inhibition on Exercise Capacity and Clinical Status in Heart Failure With Preserved Ejection Fraction. JAMA 2013; 309: $1268-1210$

9 Bonderman D, Ghio S, Felix SB et al. Riociguat for Patients With Pulmonary Hypertension Caused by Systolic Left Ventricular Dysfunction: A Phase IIb Double-Blind, Randomized, Placebo-Controlled, Dose-Ranging Hemodynamic Study. Circulation 2013; 128: $502-511$
10 Bonderman D, Pretsch I, Steringer-Mascherbauer R et al. Acute Hemodynamic Effects of Riociguat in Patients With Pulmonary Hypertension Associated With Diastolic Heart Failure (DILATE-1). Chest 2014; 146: $1274-1212$

11 Opitz CF, Hoeper MM, Gibbs JSR, et al. PreCapillary, Combined, and Post-Capillary Pulmonary Hypertension. Journal of the American College of Cardiology 2016; 68: 368-378.

12 Seeger W, Adir Y, Barbera JA et al. Pulmonary hypertension in chronic lung diseases. Journal of the American College of Cardiology 2013; 62: D109-116

13 Rosenkranz S, Ghofrani HA, Grünig E, Klose H, Olschewski H, Opitz C, Hoeper M. Pulmonale Hypertonie: Kölner Konsensus-Konferenz 2016. Dtsch Med Wochenschr 2016; 141: 1778-1782

\section{Interessenkonflikt}

C. Opitz: Keine persönlichen Einkünfte als mögliche Interessenkonflikte in den letzten 24 Monaten. Honorare an den Arbeitgeber für Vorträge und / oder Beratertätigkeiten von Actelion, Bayer, GSK und Pfizer.

S. Rosenkranz: Honorare für Vorträge und / oder Beratertätigkeiten von Actelion, Bayer, GSK, MSD, Novartis, Pfizer und United Therapeutics. Forschungsunterstützung an Institution von Actelion, Bayer, Novartis, Pfizer und United Therapeutics.

H.A. Ghofrani: Honorare für Vorträge und / oder Konsultationen von Actelion, Bayer, Gilead, GSK, MSD, Novartis, Pfizer und United Therapeutics.

E. Grünig: Honorare für Beratertätigkeiten und / oder Vorträge von Actelion, Bayer, GSK, MSD, Novartis, Pfizer und United Therapeutics. Forschungsunterstützung von Actelion, Bayer und GSK.

H. Klose: Honorare für Vorträge und / oder Beratertätigkeiten und Kongressteilnahme-unterstützung von Actelion, Bayer, MSD, GSK, UT, Novartis, OMT und Pfizer; Forschungsunterstützung von Actelion, Bayer und GSK.

H. Olschewski: Honorare für Vorträge und / oder Beratertätigkeiten von Actelion, Bayer, Belerophon, Boehringer, GSK, Menarini, MSD, Novartis, Pfizer, Roche

M.M. Hoeper: Honorare für Beratertätigkeiten und / oder Vorträge von Actelion, Bayer, Gilead, GSK, Merck und Pfizer.

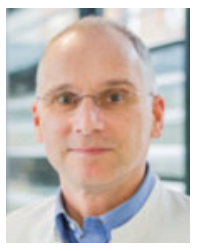

PD Dr. med. Christian Opitz ist Chefarzt der Klinik für Innere Medizin, Schwerpunkt Kardiologie, an den DRK Kliniken Berlin/Westend. c.opitz@drk-kliniken-berlin.de

Prof. Dr. med.

Stephan Rosenkranz ist Arbeitsgruppenleiter am Herzzentrum der Klinik III für Innere Medizin, Universität zu Köln.

stephan.rosenkranz@ uk-koeln.de

Prof. Dr. med. Hossein Ardeschir Ghofrani ist Oberarzt am Schwerpunkt Pneumologie der Medizinischen Klinik und Poliklinik II am Universitätsklinikum Giessen.

ardeschir.ghofrani@innere. med.uni-giessen.de

\section{Prof. Dr. med.}

Ekkehard Grünig

ist Leiter des Zentrums für PH der Thoraxklinik am Universitätsklinikum Heidelberg. ekkehard.gruenig@med. uni-heidelberg.de

\section{Dr. med. Hans Klose}

ist Leiter der Sektion Pneumologie an der II. Medizinischen Klinik und Poliklinik des Universitätsklinikums Hamburg-Eppendorf. klose@uke.de

Prof. Dr. Horst Olschewski leitet die Klinische Abteilung für Pulmonologie an der Universitätsklinik für Innere Medizin, Graz.

Horst.Olschewski@

klinikum-graz.at

Prof. Dr. med.

\section{Marius Hoeper}

ist leitender Oberarzt der Klinik für Pneumologie am Zentrum Innere Medizin der Medizinischen Hochschule Hannover.

Marius.hoeper@mhhannover.de

DOI 10.1055/s-0042-117784

Dtsch Med Wochenschr 2016; 141: 1764-1769 (c) Georg Thieme Verlag KG . Stuttgart $\cdot$ New York. ISSN 0012-0472 